\title{
Can creators and curators redefine the scientific record?
}

\author{
David Black \\ Secretary General, International Council for Science
}

There has always been a crucial linkage between creators and curators of scientific knowledge. The creators need the curators so that they can publish and establish their reputations, and the curators need the steady generation of new scientific knowledge. The advent of the internet has changed the balance of the relationship between the creators and curators, but the interdependency remains. There is also a greater call for open access to publication, and this brings a need for more appropriate controls. In this talk I shall deal with aspects of the relationship from the point of view of a researcher, and also describe steps being taken by the International Council for Science to develop a policy on open access, and the use of metrics for the assessment of quality.

Initially, I should like to say a few words about the International Council for Science (ICSU). ICSU was founded in 1931 with 41 National Members and 8 International Scientific Unions, and took over from the International Association of Academies and the International Research Council. The membership in 2014 consists of 120 National Members and 31 International Scientific Unions. The mission of ICSU is to strengthen international science for the benefit of society. One specific way in which this can be achieved is in the formulation and provision of the best available science information to guide policy making by governments and other decision makers. ICSU's vision is a world where science is used for the benefit of all, excellence in science is valued, and scientific knowledge is effectively linked to policy-making. In such an ideal world, universal and equitable access to scientific data and information would be a reality and all countries would have the scientific capacity to use these.

ICSU has a strongly multi-faceted mission to benefit society. It seeks to identify and address key issues for science and society, to foster collaboration amongst scientists across all disciplines and from all countries, to promote the participation of all scientists, regardless of race, citizenship, language, political stance, or gender, in the international scientific endeavour, and to give independent, authoritative advice to link the scientific community with governments, civil society, and industry.

ICSU has three organisational themes, namely the Universality of Science, Science for Policy, and International Research Collaboration. The boundaries between these three themes are porous, given the important linkages between them.

The Committee on Freedom and Responsibility in the conduct of Science (CFRS) is dedicated to the promotion of freedom and responsibility of scientists, as well as promoting access to data and information as a critical contribution to the conduct of science around the globe.

The Principle of Universality (freedom and responsibility) of Science maintains that the free and responsible practice of science is fundamental to scientific advancement and human and environmental 
well-being. It is based on principles of freedom, access, responsibility and anti-discrimination. There must be freedom of movement, association, expression and communication. There should also be equitable access to data, information, and other resources for research. Scientists should also take responsibility at all levels to carry out and communicate scientific work with integrity, respect, fairness, trustworthiness, and transparency. In all scientific endeavour there should be no discrimination arising from such factors as ethnic origin, religion, citizenship, language, political or other opinion, sex, gender identity, sexual orientation, disability or age.

The Principle of Universality is fully consistent with the provision of open access and open data, and ICSU is engaged in developing its policy on this and related matters. Through CFRS, it has co-sponsored with the Royal Society of London a discussion in September 2012 on the value of scientific output in the digital age. This discussion also considered the use of metrics in the assessment of the quality of scientific research. A workshop specifically on the assessment issue has also been planned by CFRS to be held in Beijing next April. The ICSU Executive Board currently has an ad hoc committee looking at open access issues, and a report is in the draft stage. While I cannot comment authoritatively on this until the report is finalised, the committee is working on acceptance of the principle of open access, such that in the interest of global science and the optimization of the benefits of science for society, access to the scientific record should be free of charge for authors and readers, it must be archived and accessible for the long-term, and it must be quality assured. When it comes to assessment, there is general agreement with the San Francisco Declaration on Research Assessment, that argues against the use of journal-based metrics as a surrogate measure of the quality of individual research articles, or to assess an individual scientist's contributions, or in hiring, promotion, or funding decisions.

ICSU plays a major role in the management of scientific data, in pursuit of its principle of equitable and universal access to data. The ICSU-World Data System is charged with the provision of long-term stewardship and quality-assessed data and data services to the international science community. Links between ICSU-WDS and the Committee on Data for Science and Technology (CODATA) are being progressively strengthened, and this should encourage a more coordinated effort linking ICSU's data policy and data management work.

The International Council for Scientific and Technical Information (ICSTI) is an Affiliate Member of ICSU. It has a membership that comprises research scientists, research libraries, primary, secondary and tertiary publishers, learned societies, data centres, governmental organisations, funding bodies, technology, software and search companies, standards organisations and scientific unions. Its affiliate relationship with ICSU brings it closer to the scientific unions and the research communities, and has also prompted interest and engagement with publishers. It is represented on ICSU's Strategic Coordinating Committee on Information and Data (SCCID) enabling it to contribute to the interaction with data centres, the management of published data and the open access to data. ICSTI also works closely with CODATA. ICSTI has established DataCite, which is an international consortium to provide easier access to scientific research data in the internet, to increase the legitimate acceptance of research data, citable contributions to the scientific record, and to support data archiving so that results can be verified and used in future research. ICSTI has recently issued a statement that encourages ICSU to approach open access on three levels: (i) open access to raw data (ii) open access to research through repositories which contain data, primary and grey literature (iii) open access to journal content including repository journals.

I would now like to make some personal remarks as a researcher, and shall comment in turn on:

- open access,

- the future landscape, 
- reputation building,

- quality assurance.

\section{Open access}

Why should there be open access? The ICSU position is based on altruism and an idealised view of the world and its scientific endeavour. Governments argue for open access because they claim this will accelerate innovation and increase productivity. There is a more pragmatic view taken by the publishers, who see this as a means to the maintenance of a viable commercial enterprise. Publishers need people to pay for their products. I have a favourite memory that is perhaps relevant here. Many years ago, at Victoria Station in London, I saw a poster advertising the supposed fact that ' $75 \%$ of top people take The Times'. Under this statement was some graffiti adding 'The other $25 \%$ pay for it'. Someone has to pay! The historical position is that funding flows from governments to research institutions, and on to researchers, who then make discoveries that require publication to the wider scientific audience. The publishers not only depend on this material, but provide some means of quality control through peer review. The authors and reviewers are not paid for their content or time, and the costs are largely related to management, printing and distribution. The advent of electronic publication significantly reduces these costs, but only if the hard copy publication ceases. Sir Robert Maxwell famously announced that the reason he went into academic publishing was because academics were the only people prepared to write for nothing. And he was correct, because academics are ego-driven and they are critically dependent on publishers to promote their results. This has led to the stratification of journals and the importance of publishing in journals that will actually be read by one's peers. This introduces factors such as support for national journals as opposed to American journals. For many years I supported the Australian Journal of Chemistry, but slowly realised that its circulation was shrinking and that it was not widely read by others in my field. On several occasions, American researchers managed to publish the same material subsequently in supposedly more impressive journals. (So much for the value of peer review and quality control.) Indeed there is a wide perception (reasonably but not entirely justified) that American researchers only read the American literature, thus driving the desire of other national researchers to publish in American journals. Academic egos are also partly responsible for the needless proliferation of journals, as editorial and editorial board positions are routinely listed on CVs as indicators of worth.

As a researcher, I confess that I have been quite happy with the old system. For many years I subscribed personally to the key journals in my field of organic synthesis. In Australia at least, the costs of such subscriptions were and still are tax deductable. The escalation of subscription costs has made the maintenance of personal subscriptions much more difficult, but the emphasis is now so heavily on access to electronic material that the hard copy is no longer necessary or even desirable. I should also point out that chemistry is perhaps an unusual area of science in relation to a general discussion. Chemistry has for many years been served (for a fee) by Chemical Abstracts, that ensured a full coverage of the area regardless of the perceived importance of journals. So publications in fringe journals (such as the Australian Journal of Chemistry) were still abstracted and therefore available to all. The abstracting process takes time, so there would always be a delay in being alerted to a paper via the abstract. The hard copy of Chemical Abstracts has now given way to the electronic SciFinder, which can be accessed from a computer (again for a fee). Our UNSW library pays for three simultaneous accesses to SciFinder, so there are times when you might have to wait to get through. In general, this is not a problem because you can log on at any time during the day or night. If this becomes a problem then more accesses could 
be bought. There are several other electronic abstracting services, but SciFinder is the most important and is universally used by chemists. It is not perfect, as Chemical Abstracts was not perfect, and misses out information from time to time. This relates to new chemical molecules which might be missed. As a chemist in the business of designing and synthesising new chemical structures, I would always check to see if certain compounds or types of structures were known, prior to the initiation of a research program to make them. With SciFinder, you can search for specific or general structures, as well as for the more usual subject, journal or author categories. So if a molecule does not appear in a SciFinder search, then it does not exist! It is clear that chemistry is already very well off in being able to access a vast amount of data very quickly. Chemistry is also perhaps unusual in that data must be provided for publications. Much of the discussion on open access places considerable emphasis on the need for data to be lodged either in publications or in repositories linked to them. This is not a major issue for chemistry, because for publication acceptance, the relevant data must be included either directly in the publication, or lodged as supplementary material that is available online.

\section{The future landscape}

Most of the discussion in the Finch Report, and other reports from the Royal Society and the European Commission are concerned with the transition from the old system to one that will be completely different. This then leads to consideration of "gold" and "green" access, and hybrid journals, and the situation is extremely complex, and for me, very confusing. I have been unable to read anything about the predicted landscape in the longer term. When I think about this, I know that I am not going to be able to grasp the situation fully, but some very strange thoughts occur to me. As a researcher, I generate new science - in my case new molecules, new structural types, with some identified new properties but with the potential for many more still to be discovered. We also generate new reactions or molecular transformations that could subsequently be used by other chemists in related work. We generate exciting advances that we would be proud of and which our peers would acknowledge as good work. But in the course of perhaps less successful work, we also generate a collection of new molecules, and these should also be reported. Perhaps these could fall into the category of "data" but they should be included in publications or repositories, so that they can be abstracted accordingly into SciFinder. What to us might be a mundane molecule might indeed be just what another chemist can use to do something completely brilliant. Also we owe it to the taxpayers who provide the research funding to publish or make this material available.

I see a far greater future emphasis on repositories as opposed to primary journals. When I think I have a story and some material that I want to tell people about, I could then send this to an appropriate repository related to my field. So there would need to be a suite of repositories based on subject codes. Various versions of such codes already exist and are widely used for the identification of reviewers for publications or research grant applications. The repositories should ideally be international and standardised, so I see a role here for international scientific unions, and consequently also for ICSU. I have no evidence to suggest that this has been considered, and it would be easier for the lead to be taken in chemistry by major national chemical societies such as the American Chemical Society (ACS) (who own SciFinder), the UK's Royal Society of Chemistry (RSC) (who own ChemSpider), or the Gesellschaft Deutscher Chemiker (GDCh), which we shall hear about next from Wolfram Koch. Publishers such as Elsevier are active in this area also, for example with Reaxys. The large national societies are also journal publishers, in the so-called "not-for-profit" category, but are really the same as the commercial publishers; they just 
spend their profits differently. I can imagine that repositories would be attractive to these large chemical societies, because they could make them freely available to members, and charge others a fee. Anyone wanting to publish in them would be attracted to become a society member, so this would be wonderful for the societies and an inexpensive and attractive alternative for the researchers, who would get many added benefits. I am a member of both the ACS and the RSC and also was for a period a member of the GDCh. There would be costs involved in the maintenance of repositories, but the process would be simpler than the current situation. Publishers could also be involved in organising repositories. However, small repositories arranged by individual universities for example, would be undesirable. It would be very helpful if there could be an agreed standard format for publication in repositories. This is where the international unions could play a major part, although they have completely failed so far to even attempt to convince publishers to agree on a standard format. Having to deal with a collection of different templates for publication is a major waste of a researcher's time. I know that publishers think they are helping, but they aren't.

The use of repositories would diminish the importance of other primary publications, which ultimately might completely disappear. However, the use of repositories would greatly increase the need for secondary publications. When you imagine the huge amount of material that will pour into each repository, there will be a pressing need to help the swamped researcher by arranging for people to sift through the material and review it. This is where the publishers could make a significant expansion in their portfolio activity, because I think that researchers would be prepared to pay for such reviews. The publishers' loss of primary publications could be replaced by a gain in secondary publications. Researchers could easily be recruited by publishers to compile reviews, as this would be a means of gaining recognition and promoting their careers. Already, the RSC is producing a small number of Specialist Periodical Reports and these could be expanded to satisfy a need.

\section{Reputation building}

As the emphasis swings to repositories of results (data) rather than primary publications, the use of the number of publications for reputation building will diminish. The future of scientific discovery will also involve greater interdisciplinary collaboration, which often leads to extended lists of authors on publications. The use of repositories is also more appropriate to deal with such collaborative approaches, as it will focus on the data rather than the authors. The quantity of publications will become less important for reputation building, as the number of authors increases. Already some universities and funding agencies request that applicants for promotion or research grants indicate for each publication their percentage contribution to the whole work. This is an impossible situation, because the supplied information cannot be validated.

So how do we then recognise the quality of the researcher and his or her contribution? Researchers already build reputations as they progress through graduate school and postdoctoral years, and this is assisted by their pedigree based on their universities and mentors. Peer opinions carry a lot of weight because researchers are regularly making presentations at conferences and become known personally within their research fields. References are much more important than publication numbers when universities are hiring new young staff members. A strong record of significant output becomes more important for promotion later on, but still the references from peers are more important. The development of journal impact factors, based on citation indices has occurred because of the computer capacity to provide them, and the idea that it helps promote some journals by giving them a veneer of quality. A reference 
citation is not an indicator of quality, so the impact factor is based on a fallacy and should never have been taken seriously. Apart from the fact that it is a poor method of assessment, this metric approach has been detrimental to the advancement of science, because it encourages researchers to join and stay in heavily populated fields, rather than striking out in an original and uninhabited area. This phenomenon is clearly evident in my own field of organic synthesis. The push for high metric scores in China and other surging Asian countries leads many researchers to work on the synthesis of known compounds using slight variations of the reaction conditions which are claimed to provide significant improvements. This tactic not only gives authors the possibility of further citations, but the work is technically easier. If you make a new compound, the collection of a lot of new data - analytical and specroscopic data is required to validate the structure, whereas a previously reported compound can be identified by simple data comparison. As a reviewer, I usually reject papers of this type, but I am clearly not a typical reviewer, as many papers describing this kind of work are published in so-called high impact journals.

There is now a wave of objection to the use of metrics for the assessment of individual quality. In 2010, Chemistry Nobel Laureate Richard Ernst, of the ETH Zürich, wrote in an article entitled "The follies of citation indices and academic ranking lists" "As an ultimate plea, the personal wish of the author remains for sending all bibliometrics and its diligent servants to the darkest omnivoric black hole that is known in the entire universe, in order to liberate academia forever from this pestilence - and there is an alternative. Very simply, start reading papers instead of merely rating them by counting citations!" More recently the San Francisco Declaration has been gaining widespread support in arguing against the use of metrics for appointment, promotion and ranking purposes. We anxiously await action by universities and funding agencies, but perhaps we should not hold our breath.

Another way in which researchers can build a reputation is through participation in international conferences. It quickly becomes obvious who are the current and rising stars of the field, who are the solid workers, and who are the unreliable strugglers. For the publishers looking for future income, conferences present an excellent opportunity, which some are already grasping. Again, in my field of chemistry, the Elsevier journal Tetrahedron sponsors annual conferences. Also the Royal Society of Chemistry is moving into the area of international conferences, in an attempt to promote their journals and look for new authors. I have no idea whether these conferences make a financial profit, but such conferences always should. There is a market issue to overcome, in that the best international chemistry conferences are those sponsored by the International Union of Pure and Applied Chemistry. Perhaps publishers will need to target specific emerging fields and create a new market model, but I think there are many opportunities available.

\section{Quality assurance}

In all the material I have read there is a totally earnest assumption that there must be quality assurance of publication. This effectively means peer review as applies currently. This view is certainly accepted officially by ICSU. I confess that I take a different personal and minority view (I suspect a minority of one). I am all in favour of peer review, but I would change the nature and timing of the process. Given the ease of internet publishing and blogging, combined with the horrifying ease of using social media (which will only become even easier into the future), I am inclined to allow researchers to submit whatever material they like. Any material sent into a repository must abide by the designated format and requirements for validation, but in my view does not need to go through the time-consuming and erratic process of peer review. The organisers of the repositories would need to provide clear instructions 
and have a minimum list of requirements, such as a basic standard of literacy and complete data compilation, for the data to be acceptable. Submissions could then easily be screened by the editorial staff, without the need to go to any external review. Currently, as a reviewer, most of my recommendations for rejection are based on the fact that there are insufficient data to provide an adequate characterisation of the new compounds described. This decision could have been taken by the editor, but here is another example of dodging the responsibility. I am an advocate of self-reviewing. If I choose to publish inferior work, then I advertise to all my peers that the work is indeed inferior. This then has connotations on my reputation. Authors of publications should be prepared to take responsibility for their work, and live by the consequences. The peer review system is undoubtedly useful in pointing out aspects of the research that could be improved or that have been overlooked. However, acceptance or rejection of a manuscript is often a very subjective issue and depends on the choice of the reviewers and their feelings at the time. Acceptance of a paper makes me feel comfortable but does not really mean that the paper is particularly worthy. As a decision-making tool, peer review is flawed. Increasingly, journals require authors to suggest reviewers, and clearly any author would suggest people who are not only relevant to the work, but at least some who are good friends and therefore likely to be supportive. In short, peer review is not necessarily related to quality control. The application of my suggested approach to the use of repositories will allow any individual scientists to make direct comments on the already accepted work, and this will become a visible peer review process. So my suggestion does not remove peer review but shifts the timing of the process. Of course if an author has committed a howler, the current system might save some embarrassment, but my suggested process would encourage authors to have manuscripts checked by cooperative colleagues before submission. Peer review after acceptance and publication could in some cases lead to further input from the authors, and in some cases lead to corrections or even retractions. This could in effect be considered to be a process of open evaluation, but would be in the form of comments, rather than in making a decision to accept or reject the publication, and of course would be entirely voluntary. Open evaluation, where reviewers are identified, has been advocated by some people for the current system, but many reviewers would prefer to remain anonymous, not necessarily because of any inhibition to make critical comments, but because of a possible reluctance to make a decision for rejection. The process of peer review after publication would certainly have an effect on an individual researcher's reputation, and would be based more on quality than quantity.

Most researchers spend a lot of time in reviewing submitted manuscripts, and I am happy to do this at my career stage, because it helps to keep me up with the literature (an impossible task). However, some complaints are emerging. Francois Diederich, a very distinguished and highly respected chemist who has recently retired after ten years as Chairman of the Editorial Board of Angewandte Chemie, has suggested that we are all refereeing ouselves to death. He argues that the peer review system is at its limit, but does not advocate its removal. He recommends tighter editorial scrutiny so that manuscripts are not sent for review unless they meet a basic standard of literacy and contain complete data. This is exactly consistent with my suggestion for repositories, although the Diederich view would proceed with the current review process, whereas I would publish and then get the peer review feedback. Professor Diederich has also noticed a serious decline in the quality of reviews, presumably because of the increasing workload, not just for publication reviews, but also reviews for research grant applications, $\mathrm{PhD}$ theses, academic promotions, and even institutions. He advocates internal assessment for $\mathrm{PhD}$ theses and academic promotions and summarises that "as a general rule, tasks and assessments that can be done within organisations on the basis of their competence and self-critical judgment, should not be moved outside". 
For many years I have taken the same view as Professor Diederich with $\mathrm{PhD}$ thesis examination, where again I am in a minority (probably of one) at UNSW. We require a thesis to be examined by two external examiners. This is usually no problem, but if the candidate and thesis are marginal, the supervisor would suggest examiners whom they know to be reasonably sympathetic. If examination was internal, then the reputations of the supervisor and the university are at stake. You have nowhere to hide. You cannot argue that distinguished external examiners thought the thesis met the appropriate standard, and therefore dodge personal responsibility. I have advocated a strict management of the candidacy from day one by an internal panel who conduct regular reviews. If the candidate is not up to standard then he or she would not be allowed to submit a thesis for the degree. Many of the top chemistry departments in the world operate in this way, and take responsibility for their $\mathrm{PhD}$ graduates.

I believe that the use of repositories will also place greater emphasis on the actual results of research in other words the data - rather than the often speculative and unjustified discussion that is frequently included in papers. This emphasis on data is something that will be much more important in the future, because modern and future computerisation enables the rational storage of vast quantities of data, which was not the case in the past.

I might digress for a moment to express my opinion that experimental data will also become much more important in the training of experimental scientists at universities. For many years, university science courses have been driven by the lecture material, which is supported by laboratory experimental work. The proportion of the latter laboratory work has steadily declined over the years, because of the high costs involved. The internet has changed things completely and future science courses must become laboratory driven. No student of chemistry needs to go to lecture courses at most universities in the world when they can stay at home in bed with a laptop or i-pad (or whatever) and study lecture courses from the top chemists in Harvard, Caltech, Cambridge or the ETH Zürich. However, they must go to university to gain training in experimental techniques. So in future the courses will be driven by the laboratory experimental work and supported by lecture material. Here is another opportunity for publishers, because I know of no text book in chemistry that defines the course in terms of an experimental program. There are many fine text books presenting material appropriate for a lecture course, and they are financial winners for publishers and authors alike. The first to enter the new format could set a new and profitable trend.

In summary, I have tried to set out how I see the future developing. Essentially I have tried to make several points:

- The use of repositories rather than primary journals.

- Repositories ideally should be controlled by international organisations and have adequate standards for acceptance of materials.

- A peer review process would take place after publication.

- Reputation building will be more dependent on personal presentations and publication quality rather than quantity.

- A switch of traditional publishing from primary to secondary journals.

It is of course an impossible task to predict what will happen because of the rapid speed of developments in communication ability and methods. For people of my vintage, struggling with computerisation, the message used to be "ask your children" - now it is "ask your grand-children".

The title of my talk was "Can creators and curators redefine the scientific record?" The answer has to be "Yes" because both groups need each other, and depend on a close collaboration. The real question is "How will the scientific record be redefined?" That remains to be seen! 